\title{
In Vivo Imaging of Intraprostatic-Specific Gene Transcription by PET
}

\author{
Frédéric Pouliot ${ }^{* 1,2}$, Breanne D.W. Karanikolas*3, Mai Johnson ${ }^{* 3}$, Makoto Sato ${ }^{3}$, Saul J. Priceman ${ }^{3}$, David Stout ${ }^{3}$, \\ Joanne Sohn ${ }^{4}$, Nagichettiar Satyamurthy ${ }^{3}$, Jean B. deKernion ${ }^{2}$, and Lily $\mathrm{Wu}^{2,3,5}$ \\ ${ }^{1}$ Institute of Urologic Oncology, David Geffen School of Medicine, University of California, Los Angeles, California; ${ }^{2}$ Department of \\ Urology, David Geffen School of Medicine, University of California, Los Angeles, California; ${ }^{3}$ Department of Molecular and Medical \\ Pharmacology, David Geffen School of Medicine, University of California, Los Angeles, California; ${ }^{4}$ Division of Laboratory Animal \\ Medicine, David Geffen School of Medicine, University of California, Los Angeles, California; and ${ }^{5}$ Institute of Molecular Medicine, \\ David Geffen School of Medicine, University of California, Los Angeles
}

\begin{abstract}
Better intraprostatic cancer imaging techniques are needed to guide clinicians in prostate cancer treatment decisions. Because many genes are specifically overexpressed in cancer cells, one strategy to improve prostate cancer detection is to image intraprostatic cancer-specific transcriptional activity. Because of the obstacles of weak cancer- or tissue-specific promoter activity and bladder clearance of many PET tracers, intraprostatic PET of gene transcriptional activity has not been previously reported. Methods: The two-step transcriptional amplification (TSTA) system that amplifies the prostate-specific antigen promoter activity was used for PET imaging of the reporter gene herpes simplex virus type-1 sr39 thymidine kinase (HSV1-sr39tk). The TSTA-sr39tk system was injected directly into prostates or prostatic tumors as a replication-incompetent adenovirus (AdTSTA-sr39tk) and imaged using PET. Results: AdTSTA-sr39tk was able to image prostate-specific antigen promoter transcriptional activity by $9-(4-18 \mathrm{~F}-$ fluoro-3[hydroxymethyl]butyl)guanine PET, in both mouse and canine prostates in vivo. Ex vivo small-animal PET images, scintigraphic counts, and sr39tk expression analysis confirmed the specificity of the observed signal. Conclusion: Here, by combining the TSTA-amplified signal with a protocol for tracer administration, we show that in vivo PET detection of transcriptional activity is possible in both mouse and immunocompetent canine prostates. These results suggest that imaging applications using transcription-based tumor-specific promoters should be pursued to better visualize cancer foci that escape detection by conventional biopsies.
\end{abstract}

Key Words: prostate imaging; localized prostate cancer; positron emission tomography; molecular imaging

J Nucl Med 2011; 52:784-791

DOI: 10.2967/jnumed.110.084582

Received Oct. 29, 2010; revision accepted Jan. 7, 2011.

For correspondence or reprints contact: Lily Wu, Institute of Molecular Medicine, Department of Molecular and Medical Pharmacology, David Geffen School of Medicine, University of California, 650 Charles E. Young Dr. S., CHS 33-118, P.O. Box 951735, Los Angeles, CA 90095-1735. E-mail: Iwu@mednet.ucla.edu

${ }^{*}$ Contributed equally to this work.

Guest Editor: Sanjiv Gambhir, Stanford University

COPYRIGHT @ 2011 by the Society of Nuclear Medicine, Inc.
$\mathbf{P}$ men in the United States (1). Treatment decision after PCa diagnosis is complicated by the variability of disease progression and the diversity of treatments available. Therefore, some patients undergo active surveillance protocols for so-called indolent cancers, whereas others undergo treatments such as radical prostatectomy for aggressive PCa (2). Indeed, pretreatment assessment of PCa risk is critical in treatment decision making. Currently, transrectal ultrasound-guided prostate biopsies are the gold standard to establish PCa diagnosis, but this technique can miss up to $48 \%$ of $\mathrm{PCa}$ foci, rendering PCa risk prediction inaccurate (3). Because of this inaccuracy, clinicians and patients can mistakenly follow minimally invasive therapy courses for aggressive diseases. On the other hand, some patients decide not to undergo minimally invasive therapies or active surveillance because of the inability to accurately predict the disease course. These facts highlight the need to improve intraprostatic assessment of $\mathrm{PCa}$ to better personalize disease treatment. Molecular PET/CT is a promising method to achieve this important task. Unfortunately, the low glycolytic activity of PCa cells and their low uptake of ${ }^{18} \mathrm{~F}$-FDG have limited the use of this common PET/CT modality in PCa imaging (4). Moreover, intraprostatic imaging is further complicated by the proximity of the prostate to the bladder, in which most water-soluble molecular tracers are excreted and cause high background signal (5). These limitations render PET-based molecular interrogation of the prostate or prostate tumors particularly challenging.

One strategy to achieve an accurate imaging signal based on the molecular signature of the cancerous tissue is to use cell-specific transcription to control expression of exogenous imaging reporter genes (6). We developed an adenovirus-based molecular imaging technique that can image functional transcriptional activity in cancer cells in vivo using PET technologies $(7,8)$. This method-the two-step transcriptional amplification (TSTA) system-has 3 components: a prostate-specific promoter, a transcription amplifier 
(Gal4VP16 fusion protein), and a PET reporter gene (herpes simplex virus type-1 sr39 thymidine kinase [HSVl-sr39tk]). The specificity of this system for prostate cells is ensured by a modified prostate-specific antigen (PSA) promoter (PSE-BC), which is active specifically in prostate-derived epithelial cells (7). Sensitivity is ensured by the TSTA configuration, a scheme that achieves an 800-fold amplification over the native PSA promoter. Finally, the reporter HSVI-sr39tk and its substrate, 9-(4-18 F-fluoro-3-[hydroxymethyl]butyl)guanine $\left({ }^{18} \mathrm{~F}-\mathrm{FHBG}\right)$, are the most widely used PET reporter gene-tracer combination (9). When incorporated into the adenoviral vector (AdTSTA-sr39tk), the TSTA system successfully expresses sufficient levels of HSV1-sr39tk for PET in several small-animal tumor models $(8,10,11)$. However, several untested challenges could limit the translation of the TSTA technology to prostate imaging in human patients. First, TSTA has not been used to image orthotopic prostate tissue by PET/CT. Second, its sensitivity and expression levels have not been tested in large, immunocompetent mammals. Finally, ${ }^{18} \mathrm{~F}-\mathrm{FHBG}$ tracer excretion through the bladder may interfere with prostate signal detection.

In this study, we established a method that allows imaging of intraprostatic, prostate-specific transcription by small-animal PET in murine prostate and prostate tumors. The feasibility of this transcription-based prostate-specific PET approach was also verified in an immunocompetent canine model. This is the first, to our knowledge, demonstration of tissue-specific transcription-based imaging by PET in the prostate of small or large immunocompetent animal models. Therefore, this report opens the develop- ment of an array of gene transcription-specific PCa imaging methods by PET that could improve pretreatment clinical staging.

\section{MATERIALS AND METHODS}

\section{Tissue Culture and Virus Production}

LAPC9 xenografts were passed continuously in male severe combined immune-deficient beige mice (Charles Rivers) and injected as a single-cell suspension (12). DU145 cells (American Type Culture Collection) were cultured in RPMI 1640 with L-glutamine (Mediatech), with 10\% fetal bovine serum. All adenoviral vectors and their production were previously described (11). Lentivirus was produced by triple transfection into HEK293T using a calcium phosphate protocol (13). The constructs pccl-cytomegalovirus (CMV)-sr39tk-internal ribosomal entry site (IRES)-enhanced green fluorescent protein (EGFP) and pcclCMV-IRES-EGFP and DU145 transductions were previously reported (14). Transduced cells were assayed for lentiviral gene expression by flow analysis on a FACScan Flow Instrument and sorted using a FACSVantage SE Sorting Flow Cytometer (BD Biosciences).

\section{Animal Work and Imaging}

Animal care and procedures were performed in accordance with the University of California Animal Research Committee guidelines. Male mice-either severe combined immune-deficient or severe combined immune-deficient/beige—were used. For Figure $1,2.5 \times 10^{5}$ DU145 cells were marked with pccl-CMV-sr39tkIRES-EGFP or pccl-CMV-IRES-EGFP lentiviruses and fluorescence-activated cell sorted before implantation. Mice were imaged with PET at day 21 after implantation, treated for $7 \mathrm{~d}$ with ganciclovir (65 mg/kg intraperitoneally, once daily), and reimaged at day 28. For Figure 2, AdTSTA-sr39tk or AdTSTA-firefly luciferase
A

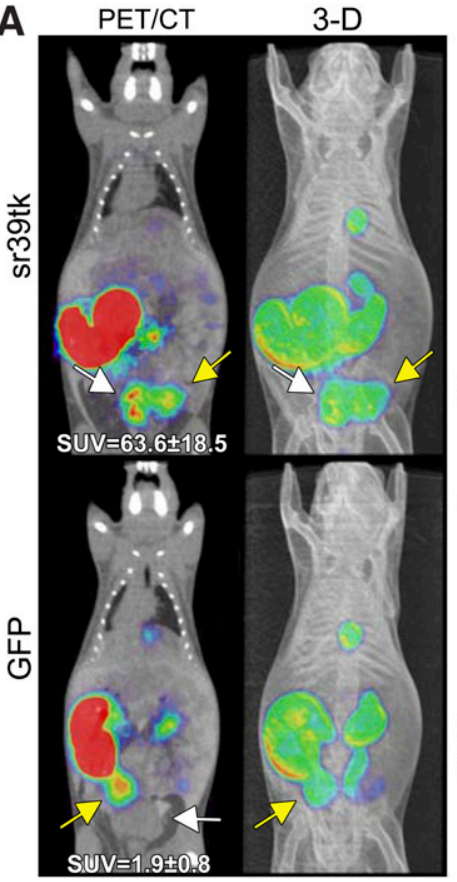

B

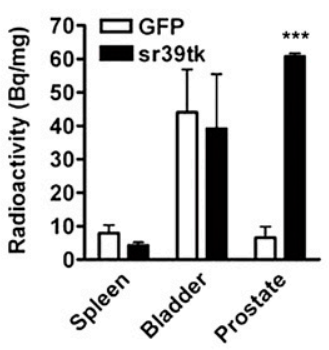

C

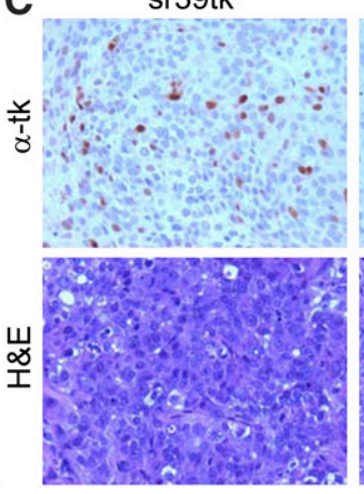

\section{GFP}

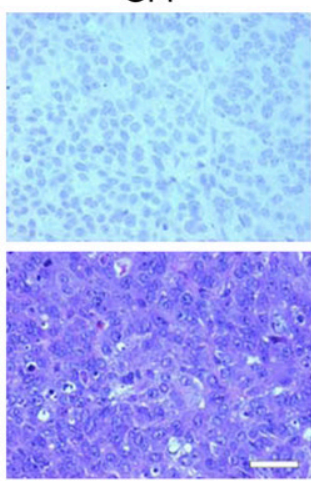

FIGURE 1. Stable expression of PET reporter HSV1-sr39tk allows detection of orthotopic $\mathrm{PCa}$ cells in vivo by small-animal PET/CT. Mice were implanted orthotopically with DU145 cells stably expressing sr39tk $(n=5)$ or green fluorescent protein $(n=5)$. All PET images were obtained using ${ }^{18} \mathrm{~F}$ FHBG. Mice were imaged at days 21 and 28 (A) to assess tumor burden by ${ }^{18} \mathrm{~F}-\mathrm{FHBG}$ uptake. In PET/CT overlay, distinct tumor signal (white arrow) is visible only in sr39tk and not green fluorescent protein tumor. In 3-dimensional (3-D) reconstruction, sr39tk tumor signal is distinct from bladder signal (yellow arrow). Quantification of ${ }^{18} \mathrm{~F}-\mathrm{FHBG}$ uptake (SUV) in orthotopic tumors on day 28 is indicated in bottom of $1 \mathrm{~A}$ (left). (B) Ex vivo quantification of ${ }^{18} \mathrm{~F}-\mathrm{FHBG}$ uptake in spleen, bladder, and prostate tumors. For $\mathrm{A}$ and $\mathrm{B}, P$ values were obtained by Student 2-tailed $t$ test, with sr39tk group compared with green fluorescent protein group. (C) Tumor immunohistochemistry against sr39tk. Scale bar = $100 \mu \mathrm{m} .{ }^{\star \star \star} P<0.001$. GFP $=$ green fluorescent protein; $\mathrm{H} \& \mathrm{E}=$ hematoxylin and eosin. 


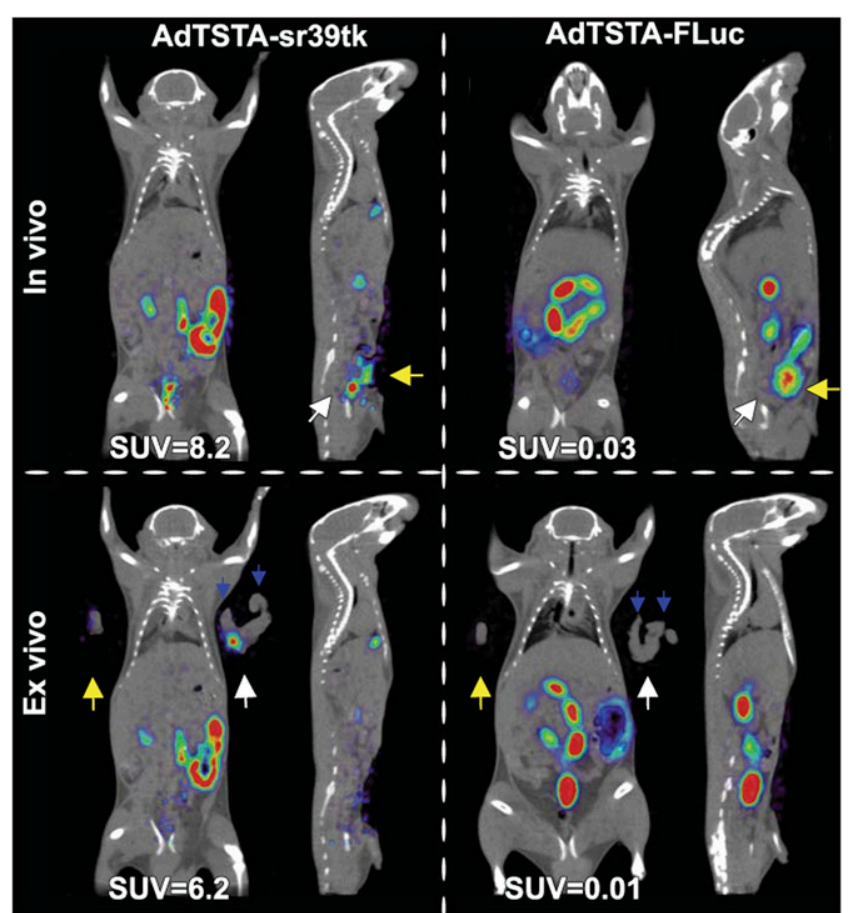

FIGURE 2. Intraprostatic delivery of AdTSTA-sr39tk allows ${ }^{18} \mathrm{~F}-$ FHBG imaging of native murine prostate. AdTSTA-sr39tk or AdTSTAFLuc was delivered to each dorsal prostate lobe via intraprostatic injection. Mice were imaged $5 \mathrm{~d}$ later. Discrete prostate signal was visible and distinct from bladder signal in sr39tk mice. Immediately after in vivo PET, organs were examined ex vivo to confirm signal localization in dorsal prostate. Quantification of prostate signals in vivo and ex vivo is reported on bottom of each panel as SUVs. White arrows = naïve prostate position; yellow arrows = bladder position; blue arrows $=$ seminal vesicles position

(FLuc) $\left(1 \times 10^{8} \mathrm{pfu}\right)$ were injected intraprostatically. For Figure 3, mice were implanted orthotopically with $2.5 \times 10^{5}$ LAPC9 cells with Matrigel (BD Biosciences). On day 14, intratumoral injections containing $1 \times 10^{9}$ pfu of AdTSTA-sr39tk or AdTSTA-FLuc were performed. For small-animal PET, mice were anesthetized using $2 \%$ isoflurane, and $7.4 \mathrm{MBq}(200 \mu \mathrm{Ci})$ of ${ }^{18} \mathrm{~F}-\mathrm{FHBG}$ were administered intravenously, followed by a 1-mL intraperitoneal bolus of saline and a 3-h tracer uptake time. A 10-min PET scan was obtained using a Focus microPET scanner, followed by a 10 min CT scan using a MicroCAT II scanner (both from Siemens Preclinical Solutions).

PET images were reconstructed using filtered backprojection to a resolution of approximately $1.8 \mathrm{~mm}$, and CT images were created using Feldkamp reconstruction of a resolution of $200 \mu \mathrm{m}$. Images were coregistered, and uptake was analyzed using AMIDE: a Medical Image Data Examiner software (15). For canine studies, male 12-mo-old purpose-bred mongrels were used (Marshall Bioresources). For all procedures, canines were anesthetized using propofol and then maintained on isoflurane for the duration of the procedures. Enrofloxacin $(5 \mathrm{mg} / \mathrm{kg}$, intravenously) was administrated before intraprostatic viral injections. For viral injections, canines were placed in left lateral decubitus position, and a rectal ultrasound probe (Sonoline G20 system with an EC94 transducer; Siemens) was used to localize the prostate, followed by a transrectal intraprostatic injection of $1 \times 10^{10}$ pfu AdTSTAsr39tk (via two $0.5-\mathrm{mL}$ injections of $5 \times 10^{9}$ ).
For PET, the canines were anesthetized and positioned in a dorsal recumbent position on an eCat HR + clinical PET scanner bed (Siemens Medical Solutions). A bladder catheter was then introduced under sterile conditions. The canines were covered with a warming blanket to maintain body temperature. A transmission scan was acquired before injection for attenuation correction. The ${ }^{18} \mathrm{~F}-\mathrm{FHBG}$ (240.5 MBq [6.5 mCi]) was administered intravenously, and a normal saline bolus $(10 \mathrm{~mL} / \mathrm{kg}$ in $1 \mathrm{~h})$ was initiated, followed by perfusion at $5 \mathrm{~mL} / \mathrm{kg} / \mathrm{h}$. The following emission PET scans were obtained: 0-60 min, dynamic scan of prostate area (axial field of view, $15.52 \mathrm{~cm}$ ); 60-92 min, whole-body scan ( 8 bed positions, $4 \mathrm{~min} /$ bed position); $120-140 \mathrm{~min}$, static scan of prostate area; and 180-200 min, static scan of prostate area. Images with a resolution of $9 \mathrm{~mm}$ were created using orderedsubset expectation maximization iterative reconstruction with scatter and attenuation correction.

Ex vivo PET was performed on prostate, right testicle, and abdominal muscle tissue in the small-animal PET scanner. For $\gamma$-ray counting, samples were analyzed in a Wizard 3" 1480 automatic $\gamma$-counter (Perkin Elmer) and then snap frozen for DNA and RNA isolation or fixed in $10 \%$ formalin for histology.

\section{DNA and RNA Extraction and Quantitative Real-Time Polymerase Chain Reaction (PCR)}

DNA was extracted using TRIzol (Invitrogen). Quantitative real-time PCR was performed using 100-150 ng of genomic DNA, $\mathrm{SyBr}$ green $2 \times$ master mix (Applied Biosystems), $10 \mathrm{nM}$ fluorescein, and $10 \mu \mathrm{M}$ each of primers for $\beta$-actin, adenovirus fiber, or sr39tk. Reactions were run on a MyiQ iCycler Real-Time PCR machine (BioRad) under the following cycling conditions: 40 repeats $95^{\circ} \mathrm{C} / 15 \mathrm{~s}, 60^{\circ} \mathrm{C} / 30 \mathrm{~s}$, and $72^{\circ} \mathrm{C} / 30 \mathrm{~s}$. Reactions were analyzed using iQ5 software (BioRad). When appropriate, samples were normalized to internal $\beta$-actin levels by the comparative cycle threshold method (16).

\section{Immunohistochemistry and Microscopy}

Paraffin-embedded tissues were processed as previously described (14). Anti-HSV1-tk antibody was used at 1:7,500 dilution (provided by Dr. Margaret E. Black). Sections were photographed using a BX41 fluorescent microscope (Olympus) fitted with a QICAM FAST 1394 camera (QImaging).

\section{RESULTS}

\section{Establishment of Small-Animal PET Protocol to Image Intraprostatic Expression of sr39tk}

Because prostate-specific PET reporter imaging is complicated by high urine background signal, we hypothesized 3 strategies to decrease ${ }^{18} \mathrm{~F}-\mathrm{FHBG}$ bladder signal: increasing tracer uptake time, increasing urine output during that uptake time, and imaging on an empty bladder. DU145 PCa cells overexpressing either HSVI-sr39tk or green fluorescent protein as a control were implanted in the prostate of immunodeficient mice (Fig. 1). Tumor cells were grown for 3 wk and imaged by small-animal PET/CT using different ${ }^{18} \mathrm{~F}-\mathrm{FHBG}$ concentrations, uptake times, and mouse hydration conditions (data not shown). Lengthening the ${ }^{18} \mathrm{~F}$ FHBG uptake time to $3 \mathrm{~h}$ (traditionally $1 \mathrm{~h}$ ), combined with hydration by intraperitoneal saline injection, achieved optimal intraprostatic sr39tk-specific small-animal ${ }^{18} \mathrm{~F}-\mathrm{FHBG}$ PET/CT signal (Fig. 1A) that was readily distinguishable 

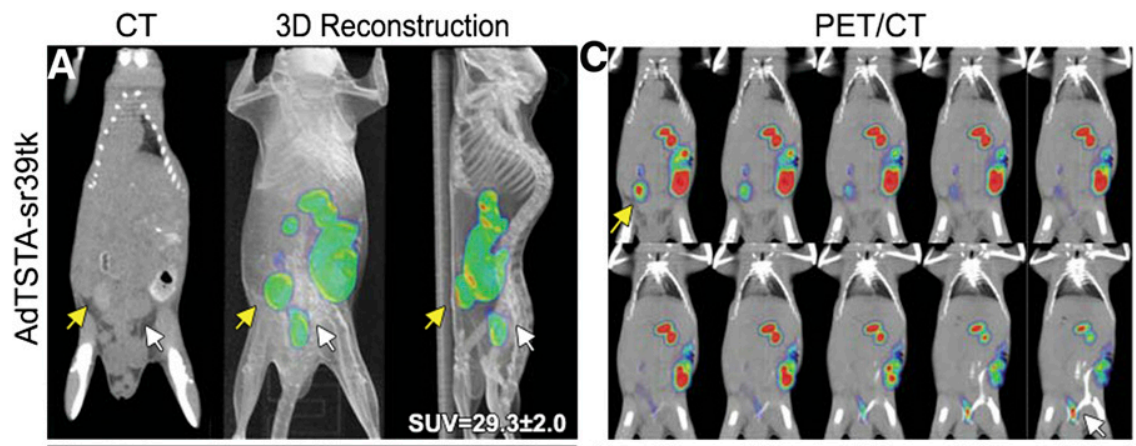

FIGURE 3. Small-animal PET/CT detection of AdTSTA-sr39tk-mediated PSA promoter transcriptional activity in orthotopic prostate tumors. Mice were implanted with LAPC9 orthotopic tumors via intraprostatic injection. After $14 \mathrm{~d}$, tumors were accessed surgically for intratumoral administration of AdTSTA-sr39tk $(n=4)($ A) or AdTSTA-FLuc $(n=4)$ (B). On days 21 (data not shown) and 28 , mice were imaged after ${ }^{18} \mathrm{~F}-\mathrm{FHBG}$ intravenous injection. Tumor signal is clear and distinct from bladder signal in sr39tk mouse. Tumor SUV quantification on day 28 represents average \pm SEM of each group ( $A$ and $\mathrm{B}$, right, $n=4$ per group, $P=0.000017$ by Student $t$ test). Sequential tomographic sections of AdTSTA-sr39tk (C) or control (D) mouse are shown from anterior to posterior (to be read left to right, top to bottom). Tumor signal (white arrow) is located inferior and posterior to bladder signal (yellow arrow). 3D = 3-dimensional.

from adjacent bladder signal (Fig. 1A). In vivo quantification of the standardized uptake values (SUVs; Fig. 1A, left) and ex vivo radioactivity measurements (Fig. 1B) confirmed the sr39tk-dependent uptake of the tracer. In fact, SUVs from sr39tk-expressing prostatic tumors were more than 30-fold higher than those from green fluorescent protein-expressing tumors $(P<0.001$, Fig. 1A). Ex vivo radioactivity counts again showed a statistically significant difference in ${ }^{18} \mathrm{~F}$-FHBG uptake in the sr39tk prostate tumors versus control tumors $(P<0.001)$ but not in other organs (Fig. 1B). Immunohistochemistry confirmed sr39tk reporter gene expression in the expected group of tumors (Fig. 1C). To further confirm the localization and specificity of the sr39tk- ${ }^{18}$ F-FHBG PET signal in the prostate tumors, we activated the suicide function of sr39tk with ganciclovir treatment $(17,18)$. The ablation of sr39tk-expressing tumor cells after ganciclovir treatment correlated with a decline of the PET tumor signal (Supplemental Fig. 1; supplemental materials are available online only at http://jnm.snmjournals. org).

\section{Intraprostatic In Vivo PET of PSA Promoter Transcriptional Activity Using Adenoviral Delivery of TSTA-sr39tk}

After the optimal conditions for PET of constitutive sr39tk expression in mouse prostate tumors were determined, the TSTA system was tested as a means of allowing PET detection of PSA promoter-mediated sr39tk expression in native mouse prostates (Fig. 2). The dorsal lobes were injected with AdTSTA-sr39tk or AdTSTA-FLuc (control) and imaged using the prostate imaging protocol. A distinct PET signal was noted at the expected prostate location (white arrow) under the bladder signal in the prostates injected with AdTSTA-sr39tk. This result confirmed the ability of AdTSTA-sr39tk to amplify tissue-specific transcription sufficiently to facilitate PET. The positive PET signal observed in the resected prostate, combined with disappearance of the pelvic signal in the mouse, confirmed that the intraprostatic photon emission is the result of AdTSTA-sr39tk-mediated PET reporter gene expression in the mouse prostate (Fig. 2, lower panels).

Next, the TSTA system was tested in an orthotopic tumor environment by injecting AdTSTA-sr39tk into an orthotopic LAPC-9 prostate tumor model (12). Six days after viral injection, mice were imaged. The PET/CT 3-dimensional reconstructions show that the orthotopic tumor is producing a pelvic signal that is distinct from the bladder signal and appears only in animals injected with AdTSTA-sr39tk (Figs. $3 \mathrm{~A}$ and $3 \mathrm{~B}$ ). Consecutive coronal 2-mm PET/CT slices demonstrate that the tumor signal position in the AdTSTA-sr39tk group is inferior and posterior to the bladder signal (Figs. 3C and 3D). In vivo, in animals injected with AdTSTA-sr39tk, prostatic signal SUVs were 18-fold higher than those in control animals injected with AdTSTA-FLuc $(P<0.001, n=4$; Supplemental Fig. 2). Taken together, these results demonstrate that intraprostatic tissue- or cancer-specific transcriptional activity can be imaged by PET.

\section{Detection of PSA Promoter Transcriptional Activity by PET in Immunocompetent Canine Model}

Although we were able to image tissue-specific transcriptional activity in immunodeficient mouse models, we proceeded to address 2 challenges that could limit the clinical translation of this transcription-based molecular imaging method. These concerns include TSTA system sensitivity in large mammals and the sufficient expression of the reporter 
gene in immunocompetent animals, given the transient nature of adenoviral infection. To mimic the clinical route of viral administration, a transrectal ultrasound probe was used to visualize the prostate and guide adenoviral injections (Fig. 4A).

Three days after adenoviral injections, the canine was imaged using a clinical PET scanner. Immediately after injection of the tracer, hydration with an intravenous saline bolus was administered to increase renal filtration. Also, to dilute residual tracer in the bladder before imaging, profuse bladder irrigation with saline was performed. At $2 \mathrm{~h}$ after injection, a focus of photon emission was detected close to the bladder neck, on the left side of the bladder (Fig. 4B).

A
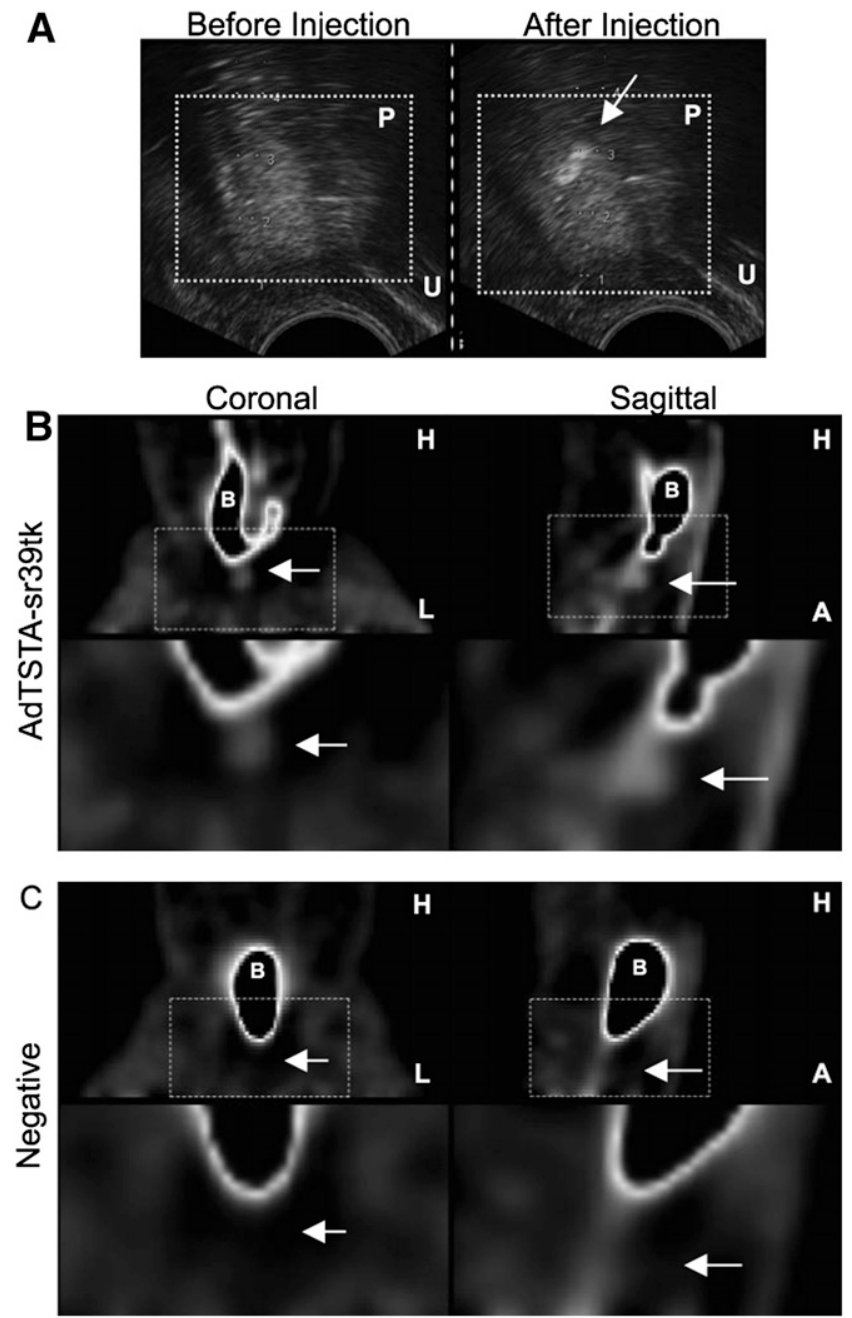

FIGURE 4. In vivo PET of prostate-specific gene transcription in canine after ultrasound-guided intraprostatic delivery of AdTSTAsr39tk. (A) Transrectal ultrasound was used to guide intraprostatic injection of AdTSTA-sr39tk into left prostate lobe. Prostate is indicated by box, and injected virus is visible as area of differential contrast (arrow). (B) At $72 \mathrm{~h}$ after injection, ${ }^{18} \mathrm{~F}-\mathrm{FHBG}$ was injected intravenously, and PET showed tracer accumulation in prostate after $2 \mathrm{~h}$. (C) PET on same canine before viral injections showed no signal in prostate area. For both $B$ and $C$, second row of images shows enlargements of areas delineated by boxes in first row. Arrows indicate prostate region. $\mathrm{A}=$ anterior; $\mathrm{B}=$ bladder; $\mathrm{H}=$ head; $\mathrm{L}=$ left side; $\mathrm{P}=$ prostate; $\mathrm{U}=$ urethra.
This focus of tracer uptake is clearly distinct from the bladder (Fig. 4B) and the urethra (Fig. 4B, sagittal view). This focus of uptake is specific to viral injection because it was not detected when the same canine was imaged $1 \mathrm{~d}$ before the viral injections (Fig. 4C).

To further demonstrate the specific uptake of tracer at the injection site, the canine prostate was imaged ex vivo, using small-animal PET/CT (Fig. 5A), revealing a focal area of photon emission in the left hemiprostate (red arrow). The injection needle tract (yellow arrow), visible on gross examination, was directed toward the left anterior prostate, which corresponds to the area of positive PET signals observed (Figs. 5A and 5B, red arrow). Tissue samples from the left prostate were further analyzed for sr39tk protein expression by immunohistochemistry. Most, but not all, of the prostatic epithelial cells in the field exhibited positive staining for tyrosine kinase expression (Fig. 5C), whereas no positive staining was observed in the area of the prostate that had not received an injection (data not shown). Scintillation counting of, and viral genome DNA analysis in, harvested organs (Fig. 5D) demonstrated significantly higher signal in the prostate than in any other tissues $(P<$ 0.001). Because of ethical considerations, we decided to curtail the use of healthy canines to a total of 2 in this study. We used the first canine to optimize the multistep procedure from sedation, ultrasound-guided vector injection, fluid management, and bladder irrigation to PET. The entire procedure was then completed for the second canine without any difficulty. Our motivation was to pursue this gene expression-based PET in a larger cohort of diseased canines soon. Despite the fact that our results were obtained in a limited number of canines, we believe these results demonstrate the feasibility of using PET to image cell-specific transcription in the prostate gland of a large immunocompetent mammal. The clinically relevant approaches taken here, such as the ultrasound-guided vector delivery and imaging with a clinical PET scanner, provide guidance to future clinical translation.

\section{DISCUSSION}

Currently in the United States, most PCas are localized and in the low-risk category at diagnosis because of increased PCa screening (19). Because the estimated median time from diagnosis to death in untreated, localized, low-risk PCas is more than $10 \mathrm{y}$, alternative managements have been proposed with the intent to limit treatment-related morbidity $(20,21)$. Among those, active surveillance and focal ablation therapy have emerged, but their use is limited by pretreatment accuracy of prostate biopsies and multifocality of PCas, showing the great need to improve pretreatment intraprostatic imaging to better identify patients suitable for alternative treatments (22).

Here, we demonstrated that tissue-specific transcriptional activity can be imaged by PET in mouse and immunocompetent canine prostates. To accomplish this study, we overcame several inherent challenges of transcription-based 
A

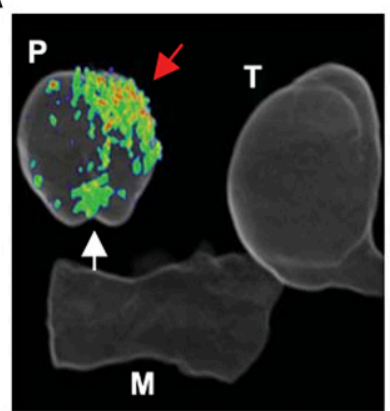

B

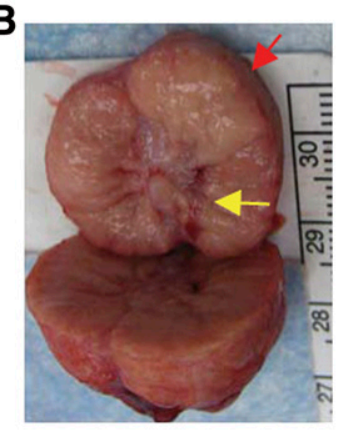

C
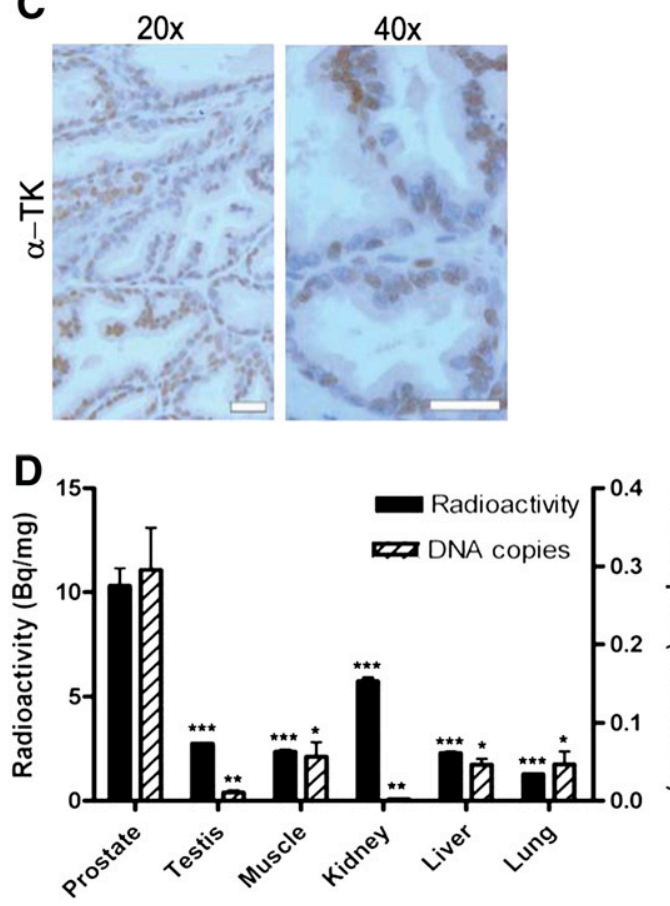

FIGURE 5. Ex vivo analysis of prostate-specific sr39tk expression after intraprostatic injection of AdTSTA-sr39tk in canine model. (A) Ex vivo imaging was performed on smallanimal PET scanner immediately after in vivo scanning to confirm ${ }^{18} \mathrm{~F}-\mathrm{FHBG}$ uptake. (B) Needle track from transrectal viral delivery was grossly visible in left anterior base of prostate (yellow arrow). For $A$ and $B$, red arrows mark position of viral injection, and white arrow marks location of urethra. (C) Immunohistochemistry against sr39tk ( $\alpha$-tk) was performed on prostate injected with AdTSTA-sr39tk. Scale bar: $50 \mu \mathrm{m}$. (D) $\gamma$-ray counting was performed on samples from various tissues to compare ${ }^{18} \mathrm{~F}-\mathrm{FHBG}$ uptake (black bars, left scale). ${ }^{18} \mathrm{~F}-\mathrm{FHBG}$ uptake in prostate is based on left hemiprostate that received vector injection. Bladder was excluded because of high background from urine contamination. DNA was isolated from tissue samples, and sr39tk levels were assayed by quantitative real-time PCR and normalized to $\beta$-actin levels (dashed bar, right scale). For $\mathrm{D}, P$ values were obtained by Student 2-tailed $t$ test, with each tissue compared with prostate. ${ }^{\star \star \star} P<0.001$. ${ }^{\star \star} P<$ 0.01 . ${ }^{*} P<0.05$. $\mathrm{M}=$ muscle; $\mathrm{P}=$ prostate; rel. $=$ relative; $\mathrm{T}=$ testis; $\mathrm{TK}=$ tyrosine kinase. prostate gland PET. First, to circumvent the relatively weak activity of tissue- or cancer-specific promoters, the TSTA method was developed to boost transcriptional activities (7). The initial studies were performed using the PSA promoter, showing that the TSTA method can augment the transcriptional activity of the PSA promoter by 800 -fold while maintaining its tissue-discriminating function (7). Subsequently, extensive use of the TSTA method by our group and others verified its ability to greatly enhance transcription of a wide range of cancer- and tissue-specific promoters, for example, PSA enhancer-PSMA enhancerTARP promoter (PPT), survivin, mucin 1, vascular endothelial growth factor, and cardiac tissue-specific promoters (23-25). Moreover, the TSTA system has been successfully adapted to optical and PET modalities $(8,11)$ and various imaging applications such as the monitoring of transcriptional factor function, cancer therapeutics (11), or metastatic events $(10,26)$. Hence, the amplification provided by the TSTA system adequately compensates for the weak activity of tissue-specific promoters and allows in vivo imaging.

A technical challenge that hinders prostate gland PET is the accumulation of water-soluble tracers in the bladder, which in turn obscures signals in the nearby prostate gland. This issue was overcome in this study by determining an optimal ${ }^{18} \mathrm{~F}$ FHBG uptake time point, which is constrained by the half-life of ${ }^{18} \mathrm{~F}$ and the excretion kinetics of ${ }^{18} \mathrm{~F}$-FHBG. The half-life of ${ }^{18} \mathrm{~F}$ is $110 \mathrm{~min}$; thus, imaging had to take place within a reasonable number of half-lives of the radiotracer. On the basis of ${ }^{18} \mathrm{~F}$-FHBG biodistribution in humans, radioactivity appears in the bladder within minutes of tracer administration and continues to accumulate up to $120 \mathrm{~min}$ (27). Because of the immediate and rapidly accumulating tracer signal in the bladder, we surmised that PET detection of prostate signals at early time points would be nearly impossible. Instead, we shifted the imaging time to later time points at 120 and $180 \mathrm{~min}$. By emptying and irrigating the bladder before imaging, bladder background was further reduced. This modified imaging protocol should be feasible to decrease bladder signal and allow intraprostatic detection of uptake of other fluorinated ${ }^{18} \mathrm{~F}$ tracers (such as ${ }^{18} \mathrm{~F}$-ethylcholine), thereby reducing clinical dependence on ${ }^{11} \mathrm{C}$-based tracers, such as ${ }^{11} \mathrm{C}$-choline and ${ }^{11} \mathrm{C}$-acetate, which require an on-site cyclotron because of the short ${ }^{11} \mathrm{C}$ half-life (28).

It is beneficial to contemplate the capabilities of the transcription-based PET technology described here with the conventional small-molecule PET tracers that reflect altered metabolic pathways in neoplasms. Unfortunately, the most widely used glucose metabolic tracer, ${ }^{18} \mathrm{~F}-\mathrm{FDG}$, is not particularly sensitive for PCa detection (4). Several new tracers for $\mathrm{PCa}$ are in late stages of clinical investigation. Among these new tracers, ${ }^{11} \mathrm{C}$-choline and ${ }^{11} \mathrm{C}$-acetate hold great promise (28). In a recent paper, Piert et al. evaluated the ability of ${ }^{11} \mathrm{C}$-choline PET/CT to detect intraprostatic PCa (29) and reported that ${ }^{11} \mathrm{C}$-choline PET could identify highgrade more precisely than low-grade PCa (Gleason score of $4+3$ and more vs. Gleason score $3+4$ and less). No significant difference in SUVs was observed between prostatic benign tissue and Gleason $6 \mathrm{PCa}$, demonstrating similar choline metabolism between well-differentiated cancers 
and benign tissue. At this juncture, a small-molecule tracer that can detect PCa specifically and sensitively remains elusive. Several other promising tracers, such as ${ }^{18} \mathrm{~F}$-dihydrotestosterone and 1-amino-3- ${ }^{18} \mathrm{~F}$-fluorocyclobutane-1carboxylic acid, still await testing in larger patient cohorts to substantiate clinical utility $(30,31)$.

We recognize that the PSA promoter-driven imaging vector used here will be unable to differentiate between malignant and benign disease because this promoter is specific not to $\mathrm{PCa}$ but to prostatic epithelial tissue. A more suitable application of the AdTSTA-sr39tk imaging technology could be to detect $\mathrm{PCa}$ local recurrence after radical prostatectomy by perianastomotic viral instillation in the absence of normal prostatic tissue or in ectopic sites of cancer dissemination such as draining lymph nodes (10). More important, our results serve as a proof of principle to deploy alternative $\mathrm{PCa}$-specific promoters that can distinguish malignant and benign disease. Several genes are upregulated at the transcriptional level specifically in $\mathrm{PCa}$, including Differential Display Code 3 (DD3/PCA3), prostate-specific membrane antigen, and $\alpha$-methylacyl-CoA racemase (32-34). Given the extensive track record of the TSTA method, it should be feasible to use the promoters of these genes to develop a parallel transcription-amplified imaging strategy to specifically image intraprostatic $\mathrm{PCa}$ foci and better guide treatments.

On the basis of our experience, an advantage of vectorbased gene imaging over conventional prostate biopsy is the increased volume of prostate tissue that can be interrogated. In this study, 2 injections of viral suspension imaged a tissue volume evaluated to be $3.8 \mathrm{~cm}^{3}$, which is far larger than the volume sampled by two 18-gauge biopsy needles. Increasing the volume of prostate assessed for cancer would, therefore, reduce the chance of missing $\mathrm{PCa}$ foci and better ascertain candidate patients for active surveillance or focal ablation therapy. Additionally, when injected into tumors, Ad-TSTAsr39tk could also detect PCa metastases in draining lymph nodes (10). Thus, the TSTA viral vector-based method could not only assess a greater volume of prostate but also investigate locoregional disease by determining lymph node involvement preoperatively.

Recombinant adenovirus is a favorable and effective vehicle to deliver genetic payloads to treat PCa (35). In particular, many recently developed vectors have incorporated molecular imaging capabilities to facilitate monitoring of transgene delivery in vivo. For instance, a well-studied approach, described by Barton et al., inserted the human sodium-iodine symporter (hNIS) gene as an imaging reporter gene in the replication-competent oncolytic adenovirus, Ad5-yCD/mutTK(SR39)rep-hNIS (36,37). Interestingly, intraprostate-directed injection of this replication-competent adenovirus achieved sufficient hNIS gene expression to image both canine and human prostates using SPECT. This oncolytic virus can selectively replicate and amplify its genome, thus boosting the expression of transgenes in cancer cells. However, use of the strong constitutive CMV promoter to control expression of the hNIS reporter gene in this virus indicates that its cancer-selective imaging capability might be negated because any transduced cells will express the imaging reporter gene. It is difficult to compare the efficacy of this oncolytic virus-directed hNIS method with the TSTA-HSV1-sr39tk-based imaging approach because of the different mechanisms of viral gene amplification, imaging reporter gene, and nuclear imaging technology. Nevertheless, the promising results demonstrated by Ad5-yCD/mutTK(SR39)rep-hNIS support the feasibility of adenovirus-mediated reporter gene imaging to interrogate cell-specific transcription directly in the prostate gland or prostate tumor, especially with the aid of transrectal ultrasound-guided vector injection.

\section{CONCLUSION}

We report the feasibility of imaging intraprostatic gene transcription using a combination of clinically available technologies. This is another step toward clinical translation of the previously described in vivo pathology concept in which tumor-specific gene promoters, gene delivery methods, reporter genes, and anatomic and nuclear imaging techniques are combined to localize and identify precisely in vivo pathologic processes, mimicking ex vivo immunohistochemistry (6). The successful demonstration of the feasibility of this imaging strategy opens the development of a more sophisticated gene transfer vehicle that could differentiate in situ benign and malignant prostate cells based on gene expression.

\section{DISCLOSURE STATEMENT}

The costs of publication of this article were defrayed in part by the payment of page charges. Therefore, and solely to indicate this fact, this article is hereby marked "advertisement" in accordance with 18 USC section 1734.

\section{ACKNOWLEDGMENTS}

This study was supported in part by NCI grants RO1 CA101904, R21 CA122693, and P50 CA092131 project 3 (to LW); the McLaughlin scholarships from Laval University; the Detweiler from the Québec Urological Association; the Canadian Institutes of Health Research fellowship scholarships (to FP); the Career Development Award from the UCLA SPORE in Prostate Cancer (P50 CA092131 to BDWK); the UCLA Tumor Biology T32 CA009056 Program (to MJ); and CDMRP Prostate Cancer Training Program (W81XWH-09-1-0538 to SJP).

\section{REFERENCES}

1. Jemal A, Siegel R, Ward E, Hao Y, Xu J, Thun MJ. Cancer statistics, 2009. $C A$ Cancer J Clin. 2009;59:225-249.

2. Klotz L. Active surveillance for prostate cancer: patient selection and management. Curr Oncol. 2010;17(suppl 2):S11-S17.

3. Bulbul MA, El-Hout Y, Haddad M, et al. Pathological correlation between needle biopsy and radical prostatectomy specimen in patients with localized prostate cancer. Can Urol Assoc J. 2007;1:264-266.

4. Machtens S, Serth J, Meyer A, et al. Positron emission tomography (PET) in the urooncological evaluation of the small pelvis. World J Urol. 2007;25:341-349. 
5. Larson SM, Schoder H. Advances in positron emission tomography applications for urologic cancers. Curr Opin Urol. 2008;18:65-70.

6. Pouliot F, Johnson M, Wu L. Non-invasive molecular imaging of prostate cancer lymph node metastasis. Trends Mol Med. 2009;15:254-262.

7. Zhang L, Adams JY, Billick E, et al. Molecular engineering of a two-step transcription amplification (TSTA) system for transgene delivery in prostate cancer. Mol Ther. 2002;5:223-232.

8. Sato M, Johnson M, Zhang L, Gambhir SS, Carey M, Wu L. Functionality of androgen receptor-based gene expression imaging in hormone refractory prostate cancer. Clin Cancer Res. 2005;11:3743-3749.

9. Min JJ, Gambhir SS. Molecular imaging of PET reporter gene expression. Handb Exp Pharmacol. 2008(185 Pt 2):277-303.

10. Burton JB, Johnson M, Sato M, et al. Adenovirus-mediated gene expression imaging to directly detect sentinel lymph node metastasis of prostate cancer. Nat Med. 2008;14:882-888.

11. Johnson M, Sato M, Burton J, Gambhir SS, Carey M, Wu L. Micro-PET/CT monitoring of herpes thymidine kinase suicide gene therapy in a prostate cancer xenograft: the advantage of a cell-specific transcriptional targeting approach. Mol Imaging. 2005;4:463-472.

12. Klein KA, Reiter RE, Redula J, et al. Progression of metastatic human prostate cancer to androgen independence in immunodeficient SCID mice. Nat Med. 1997;3:402-408.

13. Soneoka Y, Cannon PM, Ramsdale EE, et al. A transient three-plasmid expression system for the production of high titer retroviral vectors. Nucleic Acids Res. 1995;23:628-633.

14. Johnson M, Karanikolas BD, Priceman SJ, et al. Titration of variant HSV1-tk gene expression to determine the sensitivity of ${ }^{18} \mathrm{~F}$-FHBG PET imaging in a prostate tumor. $J$ Nucl Med. 2009;50:757-764.

15. Loening AM, Gambhir SS. AMIDE: a free software tool for multimodality medical image analysis. Mol Imaging. 2003;2:131-137.

16. Winer J, Jung CK, Shackel I, Williams PM. Development and validation of realtime quantitative reverse transcriptase-polymerase chain reaction for monitoring gene expression in cardiac myocytes in vitro. Anal Biochem. 1999;270:41-49.

17. Balzarini J, Bohman C, Walker RT, de Clercq E. Comparative cytostatic activity of different antiherpetic drugs against herpes simplex virus thymidine kinase gene-transfected tumor cells. Mol Pharmacol. 1994;45:1253-1258.

18. Hamel W, Magnelli L, Chiarugi VP, Israel MA. Herpes simplex virus thymidine kinase/ganciclovir-mediated apoptotic death of bystander cells. Cancer Res. 1996;56:2697-2702.

19. Ung JO, Richie JP, Chen MH, Renshaw AA, D'Amico AV. Evolution of the presentation and pathologic and biochemical outcomes after radical prostatectomy for patients with clinically localized prostate cancer diagnosed during the PSA era. Urology. 2002;60:458-463.

20. Albertsen PC, Hanley JA, Penson DF, Barrows G, Fine J. 13-year outcomes following treatment for clinically localized prostate cancer in a population based cohort. J Urol. 2007;177:932-936.

21. Bill-Axelson A, Holmberg L, Filen F, et al. Radical prostatectomy versus watchful waiting in localized prostate cancer: the Scandinavian prostate cancer group4 randomized trial. J Natl Cancer Inst. 2008;100:1144-1154.
22. Lucia MS, Epstein JI, Goodman PJ, et al. Finasteride and high-grade prostate cancer in the Prostate Cancer Prevention Trial. J Natl Cancer Inst. 2007;99: 1375-1383.

23. Figueiredo MLGS, Carey M, Wu L. Cell-specific imaging of reporter gene expression using a two-step transcriptional amplification strategy. In: Ghambir SS, Yaghoubi SS, eds. Molecular Imaging with Reporter Genes. New York, NY: Cambridge University Press; 2010:127-148.

24. Dzojic H, Cheng WS, Essand M. Two-step amplification of the human PPT sequence provides specific gene expression in an immunocompetent murine prostate cancer model. Cancer Gene Ther. 2007;14:233-240.

25. Chen IY, Gheysens O, Ray S, et al. Indirect imaging of cardiac-specific transgene expression using a bidirectional two-step transcriptional amplification strategy. Gene Ther. 2010;17:827-838.

26. Adams JY, Johnson M, Sato M, et al. Visualization of advanced human prostate cancer lesions in living mice by a targeted gene transfer vector and optical imaging. Nat Med. 2002;8:891-897.

27. Yaghoubi S, Barrio JR, Dahlbom M, et al. Human pharmacokinetic and dosimetry studies of $\left[{ }^{18} \mathrm{~F}\right] \mathrm{FHBG}$ : a reporter probe for imaging herpes simplex virus type-1 thymidine kinase reporter gene expression. J Nucl Med. 2001;42:12251234.

28. Bouchelouche K, Turkbey B, Choyke P, Capala J. Imaging prostate cancer: an update on positron emission tomography and magnetic resonance imaging. Curr Urol Rep. 2010;11:180-190.

29. Piert M, Park H, Khan A, et al. Detection of aggressive primary prostate cancer with ${ }^{11} \mathrm{C}$-choline PET/CT using multimodality fusion techniques. $\mathrm{J} \mathrm{Nucl} \mathrm{Med}$. 2009;50:1585-1593.

30. Larson SM, Morris M, Gunther I, et al. Tumor localization of $16 \beta-{ }^{18} \mathrm{~F}$-fluoro- $5 \alpha-$ dihydrotestosterone versus ${ }^{18} \mathrm{~F}$-FDG in patients with progressive, metastatic prostate cancer. J Nucl Med. 2004;45:366-373.

31. Schuster DM, Votaw JR, Nieh PT, et al. Initial experience with the radiotracer anti-1-amino-3-18 F-fluorocyclobutane-1-carboxylic acid with PET/CT in prostate carcinoma. J Nucl Med. 2007;48:56-63.

32. Zeng H, Wu Q, Li H, et al. Construction of prostate-specific expressed recombinant plasmids with high transcriptional activity of prostate-specific membrane antigen (PSMA) promoter/enhancer. J Androl. 2005;26:215-221.

33. Zha S, Isaacs WB. A nonclassic CCAAT enhancer element binding protein binding site contributes to $\alpha$-methylacyl-CoA racemase expression in prostate cancer. Mol Cancer Res. 2005;3:110-118.

34. Fan JK, Wei N, Ding M, et al. Targeting gene-virotherapy for prostate cancer by DD3-driven oncolytic virus-harboring interleukin-24 gene. Int J Cancer. 2010; 127:707-717.

35. Figueiredo ML, Kao C, Wu L. Advances in preclinical investigation of prostate cancer gene therapy. Mol Ther. 2007;15:1053-1064.

36. Barton KN, Stricker H, Brown SL, et al. Phase I study of noninvasive imaging of adenovirus-mediated gene expression in the human prostate. Mol Ther. 2008; 16:1761-1769.

37. Barton KN, Tyson D, Stricker H, et al. GENIS: gene expression of sodium iodide symporter for noninvasive imaging of gene therapy vectors and quantification of gene expression in vivo. Mol Ther. 2003;8:508-518. 